\title{
Artefakte in elektronenmikroskopischen Präparaten
}

IV. Mitt.: Dunkelfelduntersuchungen nach Fixierung mit Osmiumtetroxyd

\author{
Von Wilhelm Menke \\ Aus dem Botanischen Institut der Universität zu Köln \\ (Z. Naturforschg. 13 b, 187-189 [1958]; eingegangen am 26. November 1957)
}

\begin{abstract}
Nach Fixierung mit Osmiumtetroxyd läßt sich die Verteilung von kolloiden Kristallen in Mi. krotomschnitten im Dunkelfeld sichtbar machen. Die Kristalle werden dabei im „Licht“ ihrer Reflexe abgebildet. Bei der Beurteilung der Kontraste in Hellfeldaufnahmen müssen "auch sekundäre Veränderungen der Präparate im Elektronenstrahl berücksichtigt werden.
\end{abstract}

Kürzlich war durch Elektronenbeugungs-Diagramme nachgewiesen worden, daß bei Einwirkung von Osmiumtetroxyd in isolierten Chloroplasten kolloide, kristalline Osmiumverbindungen entstehen ${ }^{1}$. Im folgenden wird gezeigt, wie man nach Fixierung mit Osmiumtetroxyd in Mikrotomschnitten Kristalle sichtbar machen kann.

Wenn man die Objektiv-Aperturblende des Elektronenmikroskopes so weit aus ihrer axialen Stellung herausbewegt, daß keine nichtgestreuten Elektronen auf den Empfänger gelangen, so entsteht ein Dunkelfeldbild. Dieses wird durch die vom Objekt gestreuten Elektronen hervorgerufen, die bei Hellfeldbeleuchtung z. T. von der Aperturblende abgefangen werden. Da aus geometrischen Gründen nur ein Teil der gestreuten Elektronen zur Entstehung des Dunkelfeldbildes beiträgt, ist seine Helligkeit verhältnismäßig gering. Enthält das Präparat jedoch kristalline Teilchen, so reflektieren diese die auftreffenden Elektronen, wenn der Elektronenstrahl, der mit einer Netzebenenschar des Kristalls den Winkel $\vartheta$ bilde, der Bragg schen Beziehung

$$
\sin \vartheta=\frac{n \lambda}{2 d}
$$

genügt. In dieser Formel bezeichnet $d$ den Netzebenenabstand, $n$ eine ganze Zahl, die Ordnung der Reflexion, und $\lambda$ die Wellenlänge, die bei einer Be-

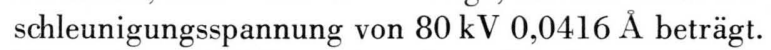
Wenn der reflektierte Strahl in die Aperturblende fällt, so kann er zur Abbildung beitragen. Die Intensität des an den Netzebenen reflektierten Strahles ist um ein Mehrfaches größer als die zur Abbildung beitragende Intensität der Elektronen, die von einem vergleichbaren amorphen Teilchen gestreut werden.

1 W. Menke, Z. Naturforschg. 12 b, 656 [1957].

* Abb. 1-7 s. Tafel S. 188 a u. b.

2 S. Strugger, Ber. dtsch. bot. Ges. 69, 177 [1956], hat eine Aufnahme eines Querschnittes durch einen Chloroplasten veröffentlicht, der an der „Umbiegestelle der Grana-Doppellamellen“" ähnliche Gebilde enthält. Nach den vorliegenden Ergebnissen besteht der Verdacht, daß es sich dabei ebenfalls um Artefakte handelt. Der Chloroplast zeigt
Daher können Kristalle in Dunkelfeldaufnahmen im "Licht" ihrer Reflexe helleuchtend auf dunklem oder diffus aufgehelltem Untergrund sichtbar gemacht werden. Da die Beugungsdiagramme von fixierten Chloroplasten gelegentlich Einkristallreflexe enthielten, die von nicht allzu kleinen Kristallen herrührten, sollten sich die bei der Fixierung entstandenen Kristalle und ihre Verteilung im Präparat direkt sichtbar machen lassen.

In Abb. $1^{*}$ ist die Dunkelfeldaufnahme eines Mikrotomschnittes durch einen vakuolisierten Chloroplasten von Allium Porrum wiedergegeben. Die Fixierung der Blattstückchen erfolgte in allen Fällen mit 1-proz. wäßriger Osmiumtetroxydlösung. Das Einbettungsmedium (Plexiglas) wurde nicht aus den Schnitten herausgelöst. Kristalle verschiedener Größe sind in großer Zahl fast regellos über den Schnitt in deutlich erkennbarem Zusammenhang mit der Struktur des Chloroplasten und des Cytoplasmas verteilt. Abb. 2 ist die zugehörige Hellfeldaufnahme. Diese wurde erst nach verhältnismäßig langer und intensiver Elektronenbestrahlung aufgenommen. Die dunklen, runden Gebilde waren ursprünglich im Präparat nicht zu erkennen. Sie sind Artefakte, hervorgerufen durch zu intensive Elektronenbestrahlung ${ }^{2}$. Auch die durch ihre Reflexe sich andeutenden Kristalle dürften in diesem Präparat schon durch Rekristallisation vergrößert sein. An Hand von Abb. 3, welche einen nachvergrößerten. Ausschnitt aus Abb. 1 darstellt, kann man ersehen, daß die anscheinend diffus aufleuchtenden Partien in Abb. 1 in Wirklichkeit zahlreiche weniger lichtstarke Reflexe enthalten. Bei Beurteilung der Dunkelfeldaufnahmen von Kristallen ist zu berücksichtigen, daß kleine Kristalle,

übrigens alle Anzeichen einer schon fortgeschrittenen Vakuolisierung und die damit verbundenen Störungen der Struktur. Durch Vakuolisierung, die in den in der vorliegenden Arbeit abgebildeten Fällen auf einem Kunstfehler bei der Fixierung beruht, können Entmischungen eintreten, die zu Bildungen führen, die dem von Strugger wiederentdeckten S e n n schen Peristromium sehr ähneln. 
die nur eine geringe Zahl von Netzebenen enthalten, auch nur geringe Intensität reflektieren und daß auf Dunkelfeldaufnahmen die Reflexe sehr kleiner Kristalle größer als die zugehörigen Kristalle sind ${ }^{3}$. Die in Abb. 4 wiedergegebene Präparatstelle enthielt nur wenige größere Kristalle. Wie aus den zugehörigen Hellfeldaufnahmen (Abb. 5) hervorgeht, zeigen die Chloroplasten Anzeichen einer beginnenden Vakuolisierung. Die Struktur des z. T. sichtbaren Mitochondrions ist stärker zerstört. Außerdem weist der Schnitt periodische Änderungen der Schnittdicke auf. Bei stärkerer Vergrößerung sah man auch hier, daß die anscheinend diffus leuchtenden Teile der Chloroplasten zahlreiche kleine Kristalle enthalten. In Abb. 6 ist die Aufnahme eines Schnittes durch einen stärker vakuolisierten Chloroplasten wiedergegeben, in dem die Verteilung der größeren Kristalle anscheinend nicht in direkter Beziehung zur Struktur des Chloroplasten steht. Mit den weiter oben erwähnten kleinen Tröpfchen dürfen größere, kugelige Einschlüsse der Chloroplasten nicht verwechselt werden, die nicht erst im Elektronenmikroskop entstehen (Abb. 7). In diesen kann man im Dunkelfeld bei etwas zu intensiver Elektronenbestrahlung zahlreiche Kristalle aufleuchten und verlöschen sehen. Dieser Effekt beruht auf einer vorübergehenden Verflüssigung des Inhaltes der Tröpfchen, der mit chemischen Umsetzungen verbunden, eine thermische Bewegung der kolloiden Kristalle bewirkt.

Daß es sich hier wirklich um Kristalle handelt, die im Licht ihrer B r a g g schen Reflexe abgebildet werden, wurde noch folgendermaßen geprüft: 1 . Verschiebung der Aperturblende bewirkt Verlöschen der einen Reflexe und Aufleuchten anderer Reflexe. Es handelt sich also nicht um diffuse Streuung. 2. Die Lage der Reflexe zum Präparat verändert sich bei Veränderung der Objektiverregung. Hierdurch ist auch eine gewisse Unsicherheit hinsichtlich der genauen Lokalisierung der Kristalle gegeben. 3. Das

3 Theoretische Grundlagen sind von C. E. Hall, Introduction to Electron Microscopy, New York, Toronto, London 1953, zusammengestellt worden.

${ }^{4}$ In der letzten Zeit scheint sich die Ansicht auszubreiten, daß bei der Erforschung der Feinstruktur von Zellen und Geweben Elektronenmikroskopie als direkte Methode den indirekten Methoden der Strukturforschung stets überlegen sei. Nur derjenige, der die den indirekten Methoden zugrunde liegenden physikalischen und chemischen $\mathrm{Zu}$ sammenhänge und Gesetzmäßigkeiten nicht übersieht, wird den Ergebnissen indirekter Methoden immer mit Skepsis begegnen. $\mathrm{Da} \beta$ auch bei einer direkten Methode, wie der Elektronenmikroskopie, die naiv morphologische Deutung nicht immer zu zuverlässigen Aussagen über das Objekt führt, ist hier nicht zum ersten Male nachgewiesen worden.
Aufleuchten der einzelnen Kristalle hängt von der Wellenlänge $a b$; Änderung der Beschleunigungsspannung bewirkt Verlöschen der einen und Aufleuchten anderer Reflexe.

Somit wurde auf zwei wenigstens z. T. voneinander unabhängigen Wegen nachgewiesen, daß bei Fixierung mit Osmiumtetroxyd in Zellen kristalline Niederschläge entstehen können. Auf beiden Wegen, nämlich durch Elektronenbeugung und Dunkelfelduntersuchung läßt sich das Vorhandensein von Kristallen eindeutig nachweisen. Im übrigen ergänzen sich die beiden Methoden ${ }^{4}$. Aus Elektronenbeugungs-Diagrammen kann man etwas über die Kristallstruktur erfahren und die chemische Natur der Kristalle ${ }^{5}$ bestimmen. Die Größe der Kristalle läßt sich in den hier in Frage kommenden Grenzen aus der Verbreiterung der Reflexe bestimmen. Im Dunkelfeld kann man die Verteilung der Kristalle direkt sichtbar machen und ihre Größe messen, wenn sie eine gewisse Größe nicht unterschreiten. Die Abmessungen kleinerer Kristalle kann man noch bei einiger Erfahrung schätzen. Die Zahl der Kristalle in einem bestimmten Bereich läßt sich jedoch nicht einfach an Hand der Aufnahmen bestimmen, da ja nur ein Teil der vorhandenen Kristalle aufleuchtet. Bei regelloser Lagerung ist jedoch die Zahl der aufleuchtenden Kristalle proportional der Zahl der vorhandenen Kristalle. Schließlich kann man an Hellfeldaufnahmen weitere Aufschlüsse über die Gestalt und Größe der Kristalle erhalten, wobei man jedoch die besonderen Effekte zu berücksichtigen hat, die auch bei Hellfeldabbildung von Kristallen durch Interferenz entstehen können ${ }^{6}$.

Kürzlich wurden auch die theoretischen Grundlagen für die Auffassung angedeutet, daß man protoplasmatische Strukturen, die vom Einbettungsmedium umgeben sind, also unter Bedingungen, wie sie in Mikrotomschnitten häufig vorliegen, wegen zu geringer Unterschiede im Streuvermögen der einzelnen

\footnotetext{
${ }^{5}$ Die chemische Natur der kristallinen Fällungen ist noch nicht ausreichend geklärt. Eine eingehendere Untersuchung der fraglichen Osmiumverbindungen und ihrer Modifikationen wird noch erfolgen. Auch können Präparate Kristalle ganz anderer Zusammensetzung enthalten, wovon man sich überzeugen kann, wenn man Präparate untersucht, die nicht mit Osmiumtetroxyd fixiert worden waren.

6 Da man so kleinen Teilchen ihren kristallinen Bau nicht ohne weiteres ansehen kann, muß dieser zunächst auf andere Weise nachgewiesen werden. Angaben über Hellfeldeffekte an Kristallen: S. LeIsEgang, Elektronenmikroskopie. Handb. d. Physik 33, 396, Berlin, Göttingen, Heidelberg 1956, und H. Raether, Elektroneninterferenzen. Handb. d. Physik 32, 443 (1957).
} 


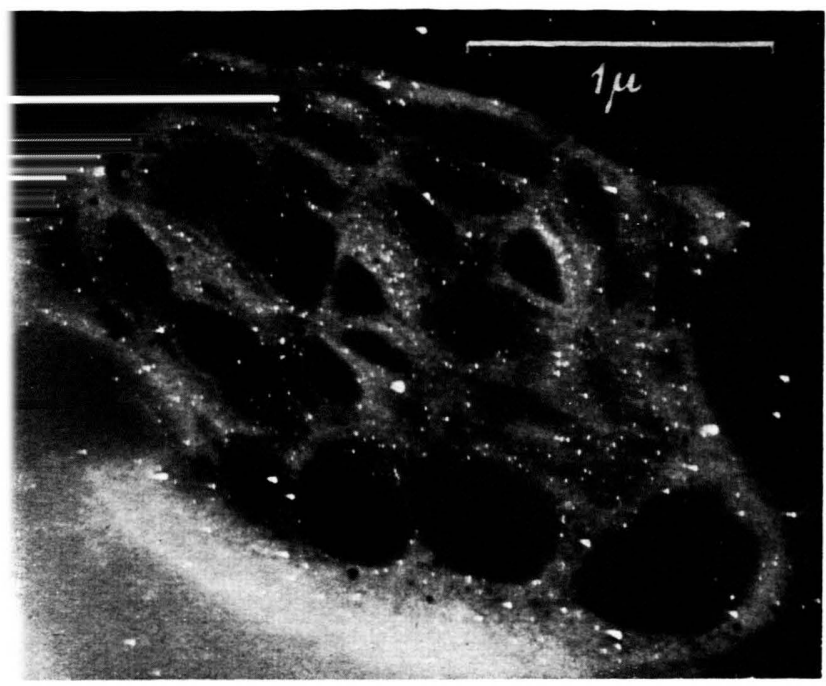

Abb. 1 .

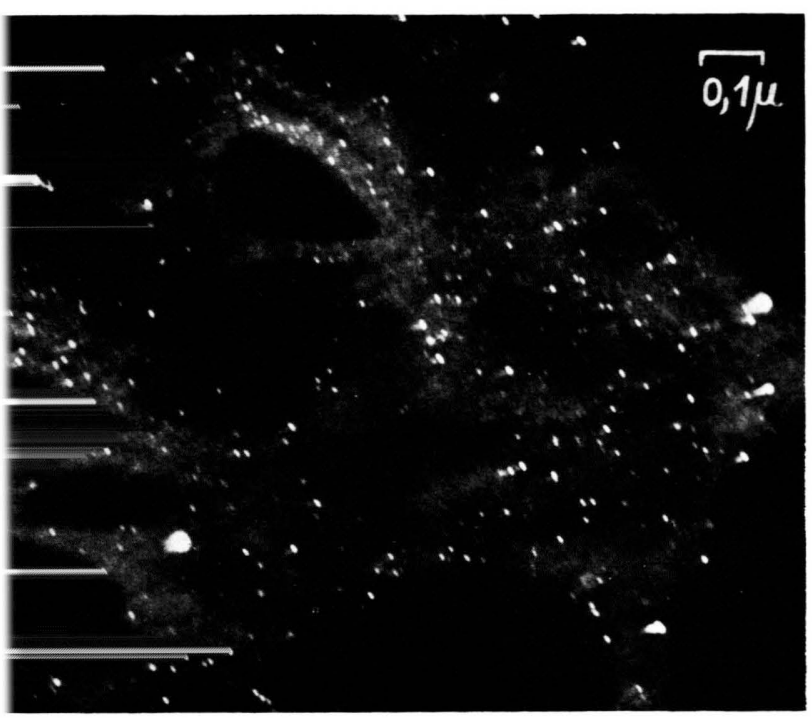

Abb. 3.

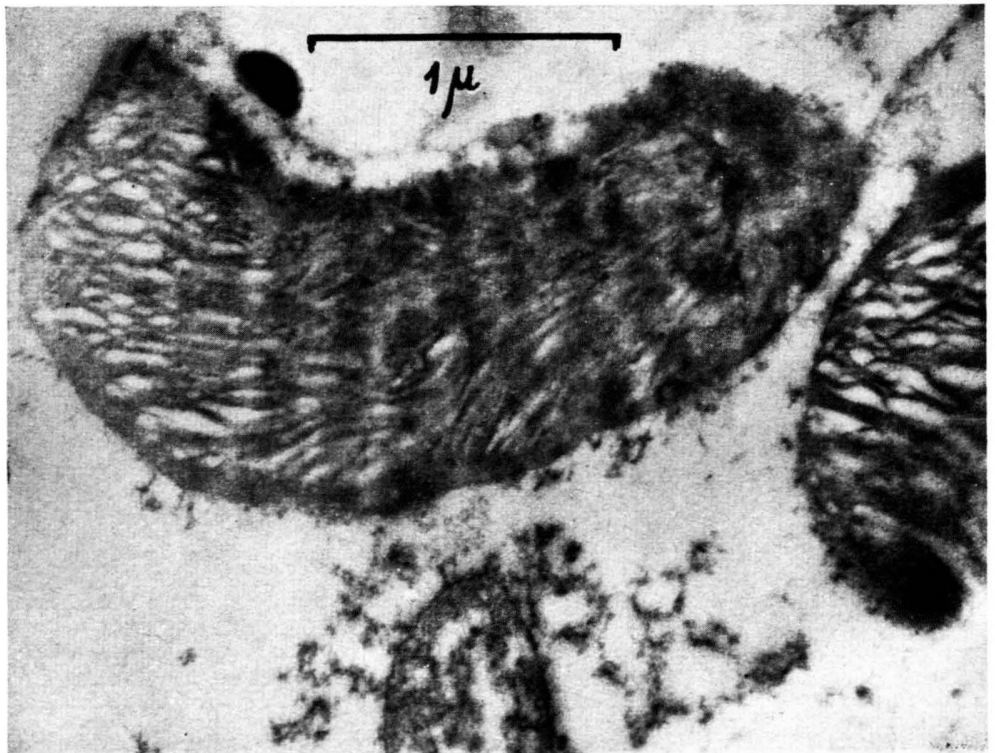

Abb. 5.

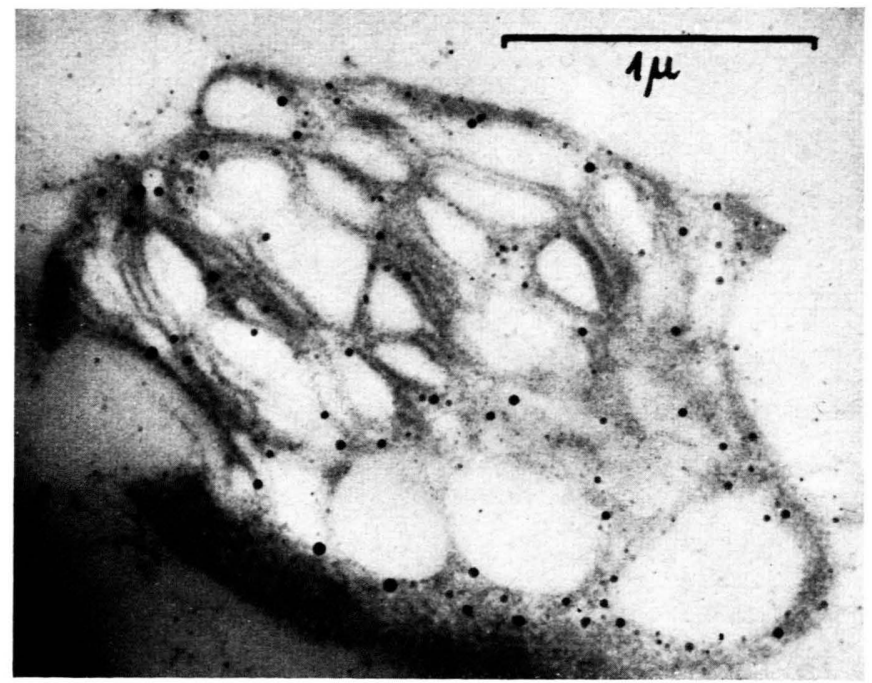

Abb. 2.

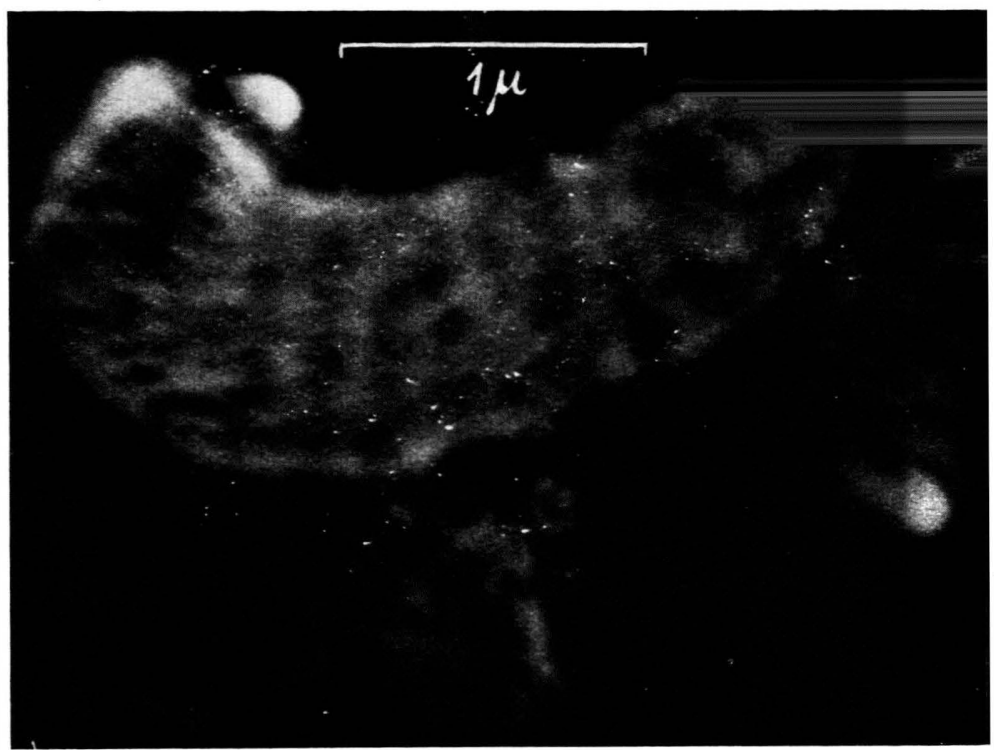

Abb. 4.

Abb. 1. Vakuolisierter Chloroplast von Allium Porrum. Im Dunkelfeld aufleuchtende Kristalle stehen in Beziehung zur Struktur des Chloroplasten.

Abb. 2. Wie Abb. 1. Hellfeldaufnahme mit Bestrahlungsartefakten.

Abb. 3. Nachvergrößerter Ausschnitt aus Abb. 1.

Abb. 4. Chloroplasten von Allium Porrum. Beginnende Vakuolisierung. Dunkelfeld.

Abb. 5. Wie Abb. 4. Hellfeldaufnahme. Am rechten Bildrand Mitochondrion mit teilweise zerstörter Struktur. 


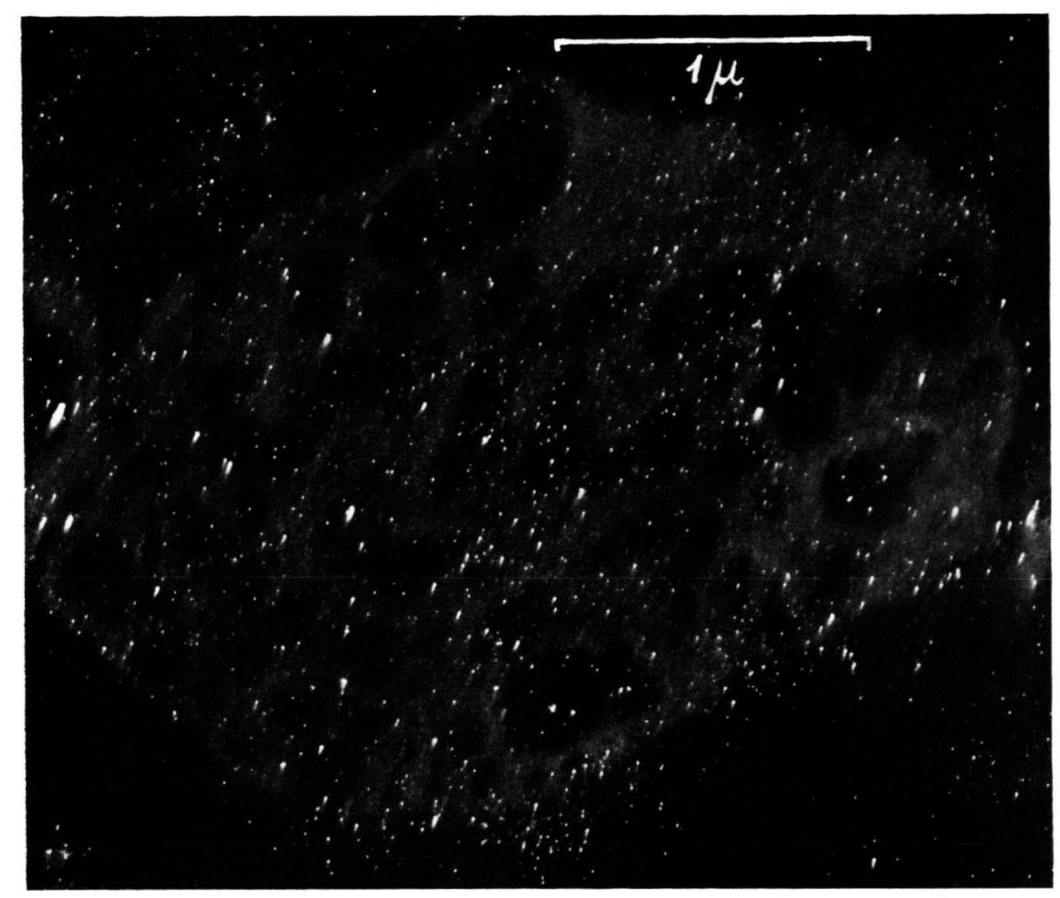

Abb. 6.

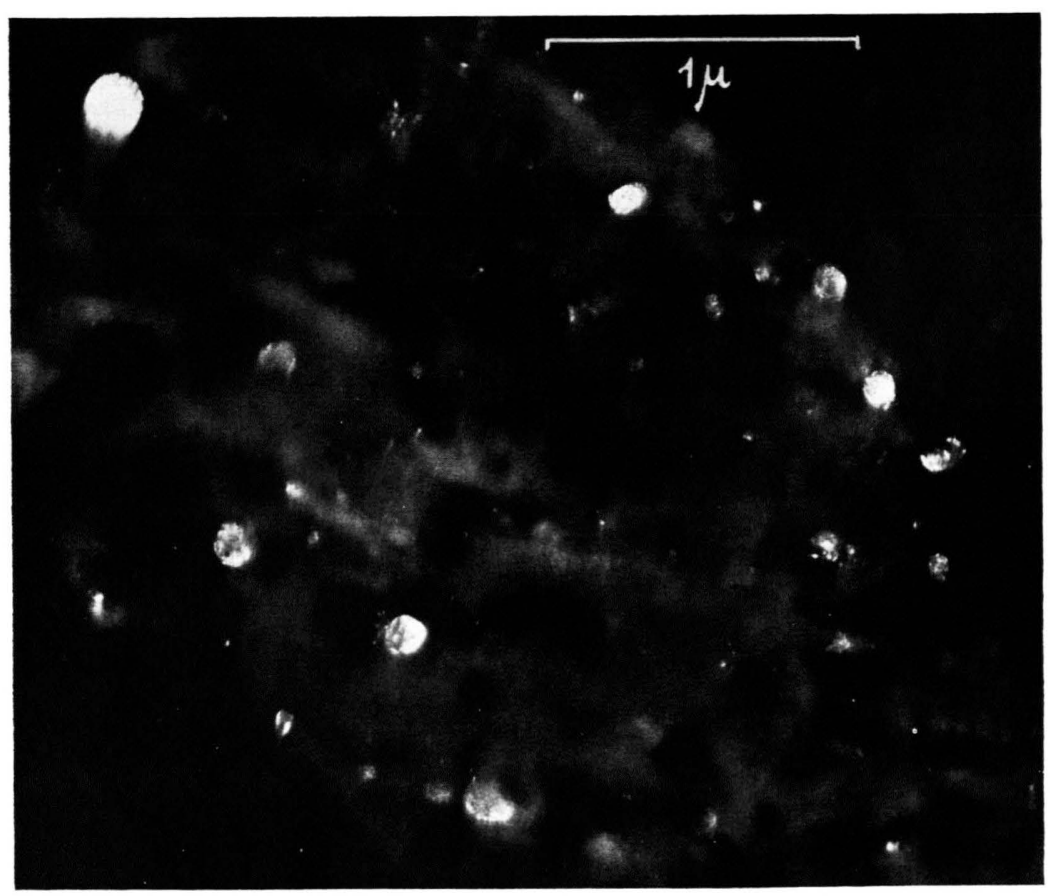

Abb. 7.
Abb. 6. Vakuolisierter Chloroplast von Allium Porrum. Verteilung der größeren Kristalle ohne Beziehung zur Struktur des Chloroplasten. Dunkelfeld.
Abb. 7. Vakuolisierte Chloroplasten von Allium Porrum mit größeren, kugeligen Einschlüssen, die zahlreiche Kristalle enthalten. 
Objektpunkte für Elektronen nicht mit ausreichendem Kontrast abbilden kann ${ }^{7}$. Zur experimentellen Überprüfung dieser Ansicht wurden Blätter von Allium Porrum durch Gefriertrocknung fixiert und in Plexiglas eingebettet. Bei den üblichen Belastungsverhältnissen ${ }^{8}$ des Präparates mit Elektronen habe ich niemals weder in dicken noch in dünnen Schnitten auch nur die Lage eines Chloroplasten erkennen können ${ }^{9}$. Zur Kontrolle vom Einbettungsmedium mit Chloroform befreite Schnitte enthielten Chloroplasten und andere Zellbestandteile. Das Ergebnis entsprach also den Erwartungen, steht jedoch im Gegensatz zu den Angaben mehrerer Autoren ${ }^{10}$, die fanden, daß man auch nach Fixierung mit Formalin und Gefriertrocknung ganz ähnliche Bilder wie nach Fixierung mit Osmiumtetroxyd erhält, selbst wenn man das Einbettungsmedium nicht extrahiert hat. Dieser Umstand beruht offenbar darauf, daß unter den Aufnahmebedingungen der genannten Autoren die Präparate während der Aufnahme weder in Plexiglas eingebettet waren ${ }^{11}$, noch aus den Baustoffen des Protoplasmas bestanden, sondern nur noch aus den nicht verdampfenden Rückständen der chemischen und thermischen Wirkungen der Elektronenbestrahlung. Diese bestehen wohl z. T. aus Kohle ${ }^{12}$. Warum unter den hiesigen Aufnahmebedingungen diese Veränderungen ausbleiben, ist mir nicht bekannt. Möglich wäre, daß die Ablagerung einer Kohlehaut ${ }^{13}$ auf dem Präparat den Dampfdruck über dem Präparat soweit erniedrigt, daß die Verdampfung gehemmt ist. Anderseits trat unter den angegebenen Belastungsbedingungen an Schnitten von Präparaten, die mit Osmiumtetroxyd fixiert worden waren, immer eine schnelle Zunahme des Kontrastes ein. Hier würde möglicherweise eine etwas stärkere Erwärmung des Präparates infolge größerer lokaler Massendicke die Verdampfung fördern und die Bildung einer schützenden Kohlehaut hinauszögern. Daß die Fixierung mit Osmium-

7 Eine ausführliche Begründung findet sich bei E. Zeitler u. G. F. BAHR, Exp. Cell. Res. 12, 44 [1957].

8 Siemens „Elmiskop I“, Kondensor 2 um 3 bis 6 Rasten des Grobeinstellers überfokussiert; Strahlstrom 5 bis $20 \mu \mathrm{A}$. Die Helligkeit des Leuchtschirm-Bildes reicht so zur Einstellung von Aufnahmen bis zu 20000 -facher Vergrößerung noch aus.

9 Auch diese Präparate enthielten Kristalle verschiedener Größe, die jedoch im Gegensatz zu den stabilen Schwermetalloxyden im Elektronenstrahl verdampfen.

10 F. S. Suöstrand, Nature [London] 171, 31 [1953] ; C. Morgan, D. H. Moore u. H. M. Rose, J. Biophys. Biochem. Cytol. 2, (Supplement), 21 [1956].

11 Eine Sublimation von Plexiglas aus den Schnitten wurde von M. L. Watson, Biochim. biophysica Acta [Amsterdam] tetroxyd zur Ablagerung größerer und feinerer kolloider, kristalliner Niederschläge in den Präparaten von Plasmastrukturen führen kann, und daß diese stärkere Kontraste in elektronenmikroskopischen Aufnahmen hervorrufen, kann wohl nicht mehr bezweifelt werden. In Fällen, in denen keine Kristalle nachweisbar sind, ist der direkte experimentelle Nachweis kolloider, amorpher Osmiumverbindungen bisher nicht erbracht worden. Wenn in dem Objekt einmal bewiesenermaßen kristallines Osmiumdioxyd entsteht, so ist nicht einzusehen, warum im anderen Fall, obwohl die bekannte Schwärzung des Präparates eintritt, überhaupt kein Osmiumdioxyd gebildet werden soll ${ }^{14}$. Viel wahrscheinlicher ist, daß im zweiten Fall amorphes Osmiumdioxyd gebildet wurde oder daß die entstandenen Kristalle so klein waren, daß sie keine Kristallinterferenzen mehr geben. Fälle, in denen die Interferenzen der Beugungsaufnahmen sehr stark verbreitert sind, wurden häufig beobachtet. Nach den Ergebnissen anderer Autoren ${ }^{14}$, die bestätigt werden konnten, spielen also beim Zustandekommen der Kontraste auch sekundäre Veränderungen der Präparate im Elektronenstrahl häufig eine Rolle. Ihr Ausmaß ist im einzelnen noch nicht abzuschätzen. Sie erschweren die Deutung der Bilder daher noch zusätzlich.

Mit den vorstehenden Ausführungen soll nicht bezweifelt werden, daß nach Fixierung mit Osmiumtetroxyd und Einbettung in Plexiglas sich gewisse Úberstrukturen der Zellorgane wie etwa die lamellaren Lipoproteidstrukturen darstellen lassen. Fraglich ist jedoch die Deutung aller noch feineren Einzelheiten wie etwa die Struktur der einzelnen Lipoproteidlamellen, weil hier Artefakte und Strukturelemente von gleicher Größenordnung nicht immer unterscheidbar sind ${ }^{15}$.

Der Deutschen Forschungsgemeinschaft bin ich für ihre großzügige Unterstützung zu Dank verpflichtet. Fräulein Helga Lehmann habe ich für ihre Hilfe bei diesen Untersuchungen ebenfalls zu danken.

10, 349 [1953], nachgewiesen. Diese tritt nach C. Morgan u. Mitarbb. ${ }^{10}$ schon bei verhältnismäßig geringer Intensität des Elektronenstrahls ein.

12 H. KönIG, Naturwissenschaften 34, 108 [1947].

13 H. König, Naturwissenschaften 35, 261 [1948].

14 L. Ornstein, J. Biophys. Biochem. Cytol. 3, 809 [1957], schreibt: „It is generally agreed that the osmium deposited in tissues probably exists in the form of mixed lower osmiumoxides and/or hydroxides and metallo-organic compounds".

15 Die Bezeichnung Elektronenfärbung ist unter diesen Umständen irreführend; denn die Durchmesser der eingelagerten Kolloidteilchen sind von gleicher Größenordnung wie die darzustellenden Strukturelemente. 